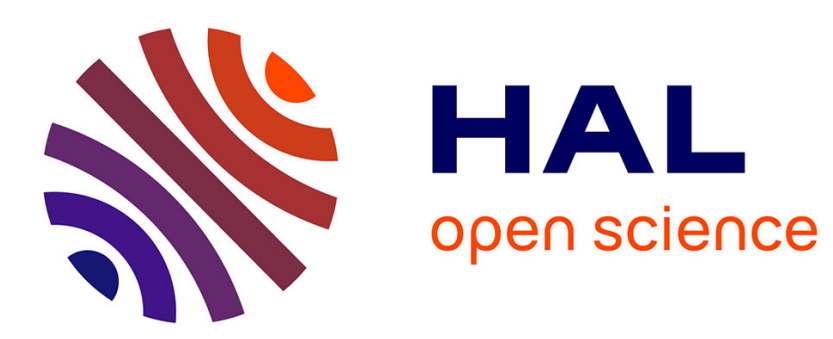

\title{
Réforme de la PAC et réduction des inégalités de revenu agricole. Premiers effets en France
}

Gilles Bazin, Alain Blogowski, Philippe Boyer

\section{To cite this version:}

Gilles Bazin, Alain Blogowski, Philippe Boyer. Réforme de la PAC et réduction des inégalités de revenu agricole. Premiers effets en France. Economie Rurale, 1996, pp.20 - 26. 10.3406/ecoru.1996.4779 . hal-02964295

\section{HAL Id: hal-02964295 \\ https://hal.science/hal-02964295}

Submitted on 12 Oct 2020

HAL is a multi-disciplinary open access archive for the deposit and dissemination of scientific research documents, whether they are published or not. The documents may come from teaching and research institutions in France or abroad, or from public or private research centers.
L'archive ouverte pluridisciplinaire HAL, est destinée au dépôt et à la diffusion de documents scientifiques de niveau recherche, publiés ou non, émanant des établissements d'enseignement et de recherche français ou étrangers, des laboratoires publics ou privés. 


\title{
Réforme de la PAC et réduction des inégalités de revenu agricole.
}

\section{Premiers effets en France}

\author{
M. Gilles Bazin, M. Alain Blogowski, Mr Philippe Boyer
}

\section{Citer ce document / Cite this document :}

Bazin Gilles, Blogowski Alain, Boyer Philippe. Réforme de la PAC et réduction des inégalités de revenu agricole. Premiers effets en France. In: Économie rurale. $N^{\circ} 232,1996$. pp. 20-26;

doi : https://doi.org/10.3406/ecoru.1996.4779

https://www.persee.fr/doc/ecoru_0013-0559_1996_num_232_1_4779

Fichier pdf généré le 08/05/2018 


\title{
Résumé
}

Les données du Réseau d'Information Comptable Agricole montrent que les disparités françaises de revenu agricole entre les différentes zones de handicaps naturels s'étaient réduites entre 1991 et 1993, soit au début de la mise en oeuvre de la réforme de la pac. En rupture avec la tendance antérieure, cette évolution était en grande partie imputable aux nouveaux mécanismes de soutien des revenus agricoles.

Ainsi, la substitution d'aides directes largement assises sur les structures (superficie, têtes de bétail) au soutien des prix, proportionnel aux quantités produites, détermine des augmentations relatives de revenu plus importantes pour les exploitations des zones de montagne ou défavorisées, moins productives à l'unité de surface. Les formes extensives d'élevage, largement représentées dans les zones difficiles, bénéficient d'aides supplémentaires substantielles qui accentuent le découplage entre la productivité de la terre et le soutien des revenus.

Toutefois, en 1994, les zones défavorisées voient s'éroder en partie leur avantage, sous l'effet des augmentations de revenus enregistrées dans les autres régions.

II semble en outre que l'évolution future des dispositifs jusqu'à la phase d'achèvement de la réforme n'aura plus d'effets marqués en termes de réductions des inégalités de revenu ; l'essentiel des mécanismes prévus ayant joué entre 1991 et 1993.

\begin{abstract}
CAP reform and reduction of income disparites. First effects in France

Based on the Farm Accounting Data Network, the analysis of french farm incomes evolution from 1991 to 1993 (first years under cap reform) shows a clear decrease of disparities bet- wen natural handicaps areas. The new mechanisms of agricultural income support and farmer's adaptations to this new deal explain this breaking off of the former trend. For instance, direct subsidies based on farm structures (acreage, cattle), instead of former production-proportional prices support, involve higher relative increases in farm incomes of mountain or less favoured areas, wich are less productive per acre. The extra subsidies granted to extensive breeding, widely present in disadvantaged areas, accentuate the decoupling between land productivity and income support. However, in 1994, the less favoured areas looses a part of their advantage, wile the other regions increases their incomes.

in the future, the evolution of cap measures up to reform completion will not have accentuated effects on incomes diparities reduction; the greater part of planned mechanisms have applied between 1991 et 1993.
\end{abstract}




\section{Déforme de la PAC et réduction des inégalités de revenu agricole Premiers effets en France}

\section{Une réforme orientée par un principe d'équité}

La réduction des inégalités de revenu agricole, qu'elles soient catégorielles ou régionales, est un des objectifs affichés de la réforme de la PAC. Dans sa préface aux propositions de la Commission en 1991, le commissaire Mac Sharry dresse, au nom de l'équité, un véritable réquisitoire contre les organisations communes de marché alors en vigueur et plaide pour une nouvelle répartition des aides aux agriculteurs européens (COMMISSION, 1991) :

«... un soutien du revenu qui repose à peu près exclusivement sur des garanties de prix est largement proportionnel au volume de production et, par conséquent, concentre la plus grande partie du soutien sur les exploitations les plus grandes et les plus intensives (...). Il en résulte que $80 \%$ du soutien assuré par le FEOGA est destiné à environ $20 \%$ des exploitations qui, par ailleurs, recouvrent la plus grande partie des terres agricoles. Le système actuel ne tient pas suffisamment compte des revenus de la grande majorité des petites et moyennes exploitations familiales ».

La nouvelle instrumentation adoptée en 1992 et ses modalités nationales d'application, bien qu'assez éloignées du radicalisme des premières propositions Mac Sharry, présentent quelques dispositifs qui vont dans le sens d'une réduction des disparités de revenus, d'une part entre grande culture et élevage bovin ou ovin, d'autre part, et pour chacune de ces orientations, entre régions intensives et bassins plus extensifs.

Il en est ainsi du mode de calcul des rendements de référence départementaux, assiette des aides compensatrices aux baisses de prix en grandes cultures, qui resserre les écarts de primes entre les départements au profit de ceux dont le rendement réel est inférieur au rendement national le rendement de référence étant égal à la moyenne entre le rendement historique départemental, pondéré de deux tiers, et le rendement moyen national, pondéré d'un tiers. Toutefois, ces primes varient fortement et un céréalier de 100 ha de la Somme percevra $250000 \mathrm{~F}$ d'aides compensatrices, tandis qu'avec la même superficie, un céréalier de la Creuse percevra un montant inférieur d'un tiers (170 000 F), ces

1. Il s'agit du chargement "PAC ", différent du chargement évalué selon les normes techniques usuelles. II est calculé en prenant en compte les animaux primables et les vaches laitières, non éligibles aux aides. Ce dispositif favorise essentiellement les systèmes allaitants. montants représentant dans les deux cas une somme équivalente au revenu des producteurs.

En élevage, divers dispositifs accentuent le soutien aux régions et aux systèmes plus extensifs en termes de chargement :

- majoration des primes bovines de $240 \mathrm{~F}$ par animal lorsque le chargement est inférieur à 1,4 UGB par hectare de superficie fourragère (1);

- plafonnement du nombre d'animaux primés à 3, UGB/ha en 1993, puis à 2 UGB/ha en 1995 (2);

- octroi d'une prime à l'herbe (300 F par ha de prairies en 1995) aux élevages dont le chargement est inférieur à 1 UGB/ha (3).

Cette dernière mesure à été instaurée en France en application du volet européen agri-environnemental d'accompagnement de la réforme. Dans son esprit, la prime à l'herbe est destinée à atténuer l'avantage relatif dont bénéficient les systèmes fourragers intensifs à base de maïs ensilage, culture non affectée par les baisses de prix programmées par la réforme mais néanmoins éligible aux aides compensatrices céréalières. Les montants totaux en cause pour chacune de ces mesures different toutefois fortement : 1,2 milliard de francs pour la prime à l'herbe contre près de 2 milliards pour le maïs fourrage en 1994 (estimation des auteurs).

\section{La mise en évidence de la logique redistributive de la réforme}

L'impact positif potentiel de la réforme sur la réduction des inégalités de revenu avait été estimé en appliquant mécaniquement (avant toute adaptation des exploitations) les nouveaux dispositifs aux structures présentés avant réforme (1991) par les exploitations françaises du réseau d'information comptable agricole (Blogowski, Boyer, 1994). Au terme de ces projections, entre 1991 et 1995 (réforme accomplie) l'excédent brut d'exploitation, EBE, moyen baisse de $19 \%$ dans les systèmes céréaliers et de $9 \%$ dans les orientations de grandes cultures, alors qu'il augmente respectivement de $9 \%$ et $18 \%$ dans les orientations bovins lait et bovins viande. La différenciation régionale de l'impact de la réforme est déterminée en grande partie par la répartition géographique de ces différentes orientations.

2. II s'agit également du chargement “PAC ". Ces plafonds sont en fait assez peu contraignants pour l'élevage bovin français (Boyer Ph., 1995).

3. Le chargement est ici calculé selon d'autres modalités - celles en vigueur pour l'éligibilité aux indemnités compensatrices de handicap naturel, comme l'indemnité spéciale montagne. 
Ainsi, les régions du nord et du centre de la France, où dominent les grandes cultures, enregistreraient des baisses moyennes de revenu allant jusqu'à $20 \%$ alors que les régions à forte spécialisation allaitante verraient leur revenu progresser nettement ( $21 \%$ en Auvergne, région qui bénéficie en outre de l'assouplissement des conditions d'éligibilité des systèmes mixtes lait-viande aux aides bovines; $17 \%$ dans le Limousin).

Ces évolutions - potentielles, puisqu'il s'agit de projections à structures inchangées - vont dans le sens d'une réduction des disparités plaine-montagne. Le revenu disponible devrait ainsi stagner en zones de plaine, alors qu'il augmenterait de $11 \%$ en zones défavorisées et de $16 \%$ en montagne.

Ainsi, l'écart moyen de revenu entre plaine et montagne devrait s'atténuer, passant de $42 \%$ en 1991 à $32 \%$ à l'horizon réforme accomplie.

Mais ces projections réalisées toutes choses égales par ailleurs ne sont qu'indicatives : il ne saurait s'agir de prévision, mais d'une évaluation de la « sensibilité » des différents systèmes ou régions aux nouveaux dispositifs. On sait désormais que les exploitations de grandes cultures, potentiellement pénalisées par les nouvelles OCM, ont connu des adaptations techniques et structurelles rapides depuis la mise en oeuvre de la réforme.

Schématiquement, l'analyse d'un échantillon constant sur 1991-93 du RICA (Blogowski, Boyer, Ronssin, 1995) montre que ces exploitations ont atténué l'impact du gel des terres en s'agrandissant : leur superficie effectivement productive ne régresse que de $5 \%$. La réduction des charges culturales - à l'hectare cultivé non gelé - et des baisses de prix de vente inférieures à celles décidées pour les prix garantis ont également contribué à maintenir le revenu moyen de ces orientations à un niveau supérieur à celui autorisé par la simple application « mécanique » du nouveau mode de soutien.

En viande bovine, la bonne tenue des cours (surtout en référence au point bas de 1991) et la poursuite de l'accroissement de la production en volume ont accentué le caractère "surcompensateur » des aides directes à l'élevage : les revenus effectifs s'avèrent également supérieurs à ceux que laissaient augurer les projections (boyer, 1995).

\section{Problématique générale de l'analyse de la réduction des inégalités de revenu au début de la réforme}

Certaines conséquences de l'application de la réforme sont appréciables dès 1993, année où les trois-quarts des aides directes prévues à l'horizon réforme accomplie sont déjà engagés (37, 5 milliards de $F$ sur environ 50 milliards de $F$ prévus pour 1995). En ce qui concerne les disparités de revenu agricole, l'année 1993 marque une inflexion par rapport à la tendance antérieure, plutôt caractérisée par l'accroissement des inégalités. Pour autant, il est extrêmement délicat, uniquement à partir d'observations empiriques, de faire la part exacte des effets conjoncturels et des incidences des nouveaux dispositifs. Ainsi, mis à par l'effet «mécanique » des aides directes, le nouveau contexte a induit à la fois des adaptations qui remodèlent durablement les structures des exploitations (agrandissement, par exemple), et des réactions plus conjoncturelles sur la pérennité desquelles on peut s'interroger : les réductions d'intrants observées en grande culture seront-elles durables? (Bazin, Carles, Kroll, 1995).
Malgré ces difficultés, cet article se propose d'analyser les premières tendances en matière de réduction des disparités de revenu agricole, d'évaluer l'effet réforme dans ces évolutions, et de s'interroger sur le caractère durable de cette nouvelle situation.

L'analyse a été conduite à partir des données du RICA de 1991, 1993 et 1994. Compte tenu de l'importance des disparités existant entre les zones de handicaps géographiques - plaine, zones défavorisées, montagne - et de l'objectif de réduction des inégalités de revenu affichée par la réforme, ce zonage restera au centre de notre approche. S'agissant tout d'abord de rendre compte de la nouvelle répartition du revenu agricole entre les zones de handicaps, les évolutions seront retracées sur la base des échantillons complets annuels (1991, 1993 et 1994) du RICA. Elles découlent donc à la fois des adaptations individuelles des exploitations pérennes et des variations de la démographie agricole.

\section{La réduction des disparités entre zones de handicaps en 1993 et 1994}

Les courbes d'évolution des revenus et des aides selon les zones de handicaps montrent bien la rupture due à la réforme de la PAC en 1993 et 1994. En fait, l'augmentation des aides directes débute en 1992, avec l'instauration de primes compensatrices pour les oléagineux, et s'amplifie en 1993 avec la poursuite de la réforme.

\section{Graphique 1. Evolution du revenu (résultat courant) et des aides selon les zones de handicaps}

\section{Illustration non autorisée à la diffusion}

\section{Source : RICA}

Alors qu'entre 1984 et 1991 les différences de revenus entre les zones de montagne et défavorisées et les zones de plaine restent comprises entre $40 \%$ et $50 \%$, les années 1993 et 1994 marquent des évolutions nettement différentes. De 1991 à 1993, les revenus augmentent plus fortement dans les zones de montagne $(+37 \%$ pour le résultat courant), et surtout dans les zones défavorisées $(+47 \%)$, que dans les zones de plaine $(+1 \%)($ cf. tableau 1$)$. 
On assiste ainsi à un rapprochement des revenus moyens des différentes zones. En 1993, les différences ne sont plus que de $26 \%$ entre la montagne et la plaine, et de $15 \%$ entre les zones défavorisées et la plaine, ce qui est considérable compte tenu de l'évolution antérieure (Bazin, 1993).

Si l'année 1994 ne renverse pas les tendances à l'augmentation des revenus dans les zones de montagne $(+12 \%)$ et défavorisées $(+14 \%)$, elle marque cependant de nouveau un accroissement des disparités plaine-montagne du fait de la plus forte hausse enregistrée dans les régions de plaine $(+27 \%$ entre 1993 et 1994). Cette progression est due pour une large part à la bonne tenue des cours des céréales et des oléagineux, ainsi qu'à l'accroissement de $25 \%$ des aides perçues par exploitation - contre $19 \%$ en montagne et $17 \%$ dans les zones défavorisées. En conséquence, par rapport aux zones de plaine, le résultat courant en 1994 reste inférieur de $34 \%$ en montagne et de $24 \%$ en zones défavorisées. Après le net resserrement des revenus constaté en 1993, l'évolution est de nouveau divergente, même si la réduction des disparités par rapport à la période antérieure reste appréciable : de l'ordre de 10 points.

En plaine, les aides directes représentent $47 \%$ du résultat courant en 1994. Elles sont ainsi pratiquement multipliées par 5 par rapport à 1991. Cette forte augmentation n'implique cependant pas que le soutien global des producteurs ait augmenté d'autant. Elle ne fait que traduire la substitution partielle de versements directs au soutien précedemment accordé par des prix garantis, et assuré par d'autres transferts : coût budgétaire des restitutions aux exportations, et surcoût d'approvisionnement supportés par les clients du secteur.

Le niveau d'aides le plus élevé est atteint en zones défavorisées avec $114300 \mathrm{~F}$ par exploitation, soit $82 \%$ du revenu. Compte tenu de leurs orientations de production, ces zones perçoivent des aides importantes aux grandes cultures (66 100F par exploitation) et les plus forts montants d'aides à l'élevage avec $35600 \mathrm{~F}$ de primes bovines et ovines par exploitation. Elles bénéficient nettement de la réforme, en concentrant $22 \%$ des aides en 1994 , pour $17 \%$ de l'ensemble des exploitations.

Les zones de montagne sont peu touchées par le nouveau régime céréalier et étaient déjà soutenues avant réforme par des aides directes : ces dernières progressent donc moins qu'ailleurs $(+90 \%$ entre 1991 et 1994).

Avec $79600 \mathrm{~F}$ par exploitation, elles représentent $67 \%$ du revenu des exploitations. Mais la composition de ces aides est modifiée.

Alors que les indemnités compensatrices de handicaps représentaient près de la moitié des subventions aux exploitations de montagne en 1991, elles en représentent moins du tiers en 1993 et seulement le quart en 1994.

Les exploitations de montagne, qui constituent $16 \%$ de l'univers du RICA, concentraient près de $30 \%$ des aides directes en 1991, elles en perçoivent moins de $15 \%$ en 1994.

On peut dire que la généralisation du soutien par des aides directes atténue la spécificité de la politique de la montagne, ce qui ne manque pas d'inquiéter les professionnels de ces régions.

\section{Illustration non autorisée à la diffusion}

Source : RICA

Au-delà de ces résultats moyens différenciés selon les zones, l'analyse de la répartition des revenus individuels confirme la réduction des inégalités.

Le critère ci-après utilisé pour étudier la redistribution des revenus de 1991 à 1994 entre les exploitations est l'écart à l'EBE moyen : chaque exploitation est caractérisée par l'écart relatif que présente son excédent brut d'exploitation avec l'EBE moyen national de l'année considérée, exprimé en pourcentage.

L'écart est ainsi négatif, égal à 0 , ou positif pour une exploitation dont l'EBE est respectivement inférieur, égal, ou supérieur 
à la moyenne nationale. Un écart de $100 \%$ correspond à un EBE double de la moyenne nationale.

Toutes zones de handicaps naturels confondues, la courbe de répartition des revenus individuels se resserre légèrement autour de la moyenne entre 1991 et 1994.

Cette évolution globale assez peu marquée résulte du poids relativement important des exploitations de plaine, pour lesquelles la courbe de répartition est peu modifiée.

L'évolution est plus sensible en zones défavorisées et de montagne. Pour les exploitations de ces régions, la courbe de répartition des revenus rapportés à la moyenne nationale toutes zones se modifie sensiblement (graphique 2).

Alors que plus de $80 \%$ des exploitations des zones défavorisées ou de montagne présentaient un EBE inférieur à la moyenne nationale en 1991, moins des trois quarts se trouvent dans ce cas en 1993. Les premières années de la réforme se caractérisent donc par une certaine redistribution des revenus en faveur des zones défavorisées et de montagne.

Toutefois, le gain relatif de ces régions s'érode quelque peu en 1994. En plaine, les exploitations de grandes cultures ont en effet poursuivi leurs adaptations (agrandissement, économies de charges), atténuant les effets redistributifs de la réforme. En outre, une partie du resserrement observé en 1993 était dû à la conjoncture défavorable qui avait affecté certaines productions de plaine en 1991 - fruits et légumes.

\section{Graphique 2. Fréquences cumulées des exploitations des zones défavorisées et de montagne selon leur écart à l'EBE moyen national}

\section{Illustration non autorisée à la diffusion}

\footnotetext{
Source : AICA - Echantillon complet
}

Malgré la réduction des disparités constatées, le degré de concentration des revenus reste très élevé puisque moins de 100000 exploitations se partagent la moitié du revenu total en 1993 et 1994.

\section{Des évolutions contrastées selon les orientations de productions et les zones de handicaps}

Ces redistributions de revenus sont en partie imputables à l'impact différencié de la réforme selon les orientations de production, lesquelles sont inégalement représentées dans les différentes zones.
Les orientations technico-économiques, OTEX, définies par la statistique agricole ont été ici reclassées dans quatre grands agrégats : Cultures, prédominance de productions végétales visées par la réforme; Elevage, élevage d'herbivores, également concerné par la réforme; Mixte, combinaison des productions animales et végétales précédentes; Autres, peu de productions soumises aux nouveaux dispositifs : cultures spéciales, élevage hors-sol...

Tableau 2. Evolution de l'EBE moyen par orientation et zone. $\Delta \%$ : variation 1991.94

Illustration non autorisée à la diffusion

Source : RICA - Echantillon complet. Francs courants par exploitation

\section{Les orientations de productions végétales visées par la réforme}

Le resserrement des écarts de revenus constaté entre 1991 et 1993 ne perdure pas en 1994 (graphique 3).

Graphique 3. Ecart à l'EBE moyen national - "Cultures "

\section{Illustration non autorisée à la diffusion}

Source : RICA- Echantillon complet

Entre 1991 et 1993, l'EBE moyen des zones défavorisées augmente nettement plus qu'en plaine ( $22 \%$ contre $8 \%$ ). Cette évolution constatée au début de la mise en oeuvre de la réforme est la résultante de deux effets :

- d'une part, l'agrandissement moyen des exploitations de grandes cultures est plus important sur cette période en zones défavorisées qu'en plaine ( $23 \%$ contre $13 \%$ );

- d'autre part, le revenu moyen à l'hectare diminue moins sur cette période en zone défavorisée qu'en plaine ( $1 \%$ contre $5 \%$ ).

Ce second effet est en partie dû au découplage des aides instaurées par la réforme. En présentant des rendements moins élevés, les exploitations des zones défavorisées enregistrent moins de pertes de produit à l'hectare sous l'effet des baisses 
de prix. La couverture de ces pertes par les aides directes s'avère meilleure qu'en plaine, comme le montre le tableau cidessous.

\section{Tableau 3. Produit et aides par hectare dans les exploitations de " cultures"}

\begin{tabular}{lrr}
\hline & Plaine & $\begin{array}{r}\text { Zones } \\
\text { défavorisées }\end{array}$ \\
Produit F/ha 1991 & 10150 & 7615 \\
Produit F/ha 1993 & 7515 & 5300 \\
Perte de produit F/ha & -2635 & -2315 \\
Aides F/ha 1991 & 170 & 285 \\
Aides F/ha 1993 & 1660 & 1865 \\
Gain d'aides F/ha & 1490 & 1580 \\
Solde F/ha & -1145 & -735 \\
\hline
\end{tabular}

Toutefois, entre 1993 et 1994, la situation des exploitations de grandes cultures se dégrade en zones défavorisées et s'améliore en plaine, et l'écart de revenu observé avant réforme se reconstitue. Sur cette période, la SAU moyenne des exploitations de plaine augmente encore $(+3 \%)$, tandis qu'elle diminue ailleurs $(-3 \%)$. En outre, l'EBE par hectare augmente de $10 \%$ sur 1993-94 en plaine, contre 7\% en zones défavorisées. Cette différence est essentiellement imputable à une baisse du produit par hectare en zones défavorisées $(-7 \%)$, liée à une diminution de rendement $(-8 \%$ en moyenne pour la production de l'hectare de SCOP).

\section{L'élevage d'herbivores}

Dans toutes les zones, l'EBE de l'orientation Elevage était inférieur à l'EBE moyen national en 1991 (graphique 4). Cette situation s'atténue en 1993 et 1994 : les revenus de chaque zone se rapprochent de la moyenne.

Le résultat de cette évolution apparaît toutefois contrasté selon les zones. Ainsi, les élevages de plaine atteignent dès 1993 un revenu très proche de la moyenne nationale, tandis que les zones défavorisées et surtout la montagne restent très en retrait.

En plaine, la progression du revenu entre 1991 et 1994 est imputable à la fois à l'agrandissement $(+19 \%$ en SAU) et à l'amélioration de l'EBE à l'hectare $(+16 \%)$, essentiellement due à l'augmentation des aides (+ $590 \mathrm{~F}$ par ha).

\section{Graphique 4. Ecart à l'EBE moyen national - Orientation "Elevage"}

Illustration non autorisée à la diffusion
L'EBE moyen des élevages des zones défavorisées progresse très fortement $(+52 \%)$, sous l'effet de l'agrandissement $(+$ $11 \%$ ) et d'une forte amélioration de l'EBE à l'hectare. Ce dernier a augmenté de $37 \%$, dont deux tiers sont dus à l'augmentation des aides (+ $630 \mathrm{~F}$ par ha), le reste étant imputable à la croissance du produit et à la réduction des charges par hectare.

En montagne, l'EBE à l'hectare a progressé plus faiblement $(15 \%)$, une chute de la production par hectare ayant atténué l'impact de la croissance des aides directes $(+510 \mathrm{~F}$ par ha).

En plaine, les aides directes rapportées à l'hectare font plus que doubler de 1991 à 1994. Les élevages de ces régions sont davantage orientés vers la production laitière - près de $60 \%$ du produit total en 1993 - et présentent des chargements plus élevés (1,6 UGB/ha SFP) qu'en montagne ou qu'en zones défavorisées. Ils sont donc moins souvent éligibles aux primes bovines ou ovines revalorisées par la réforme et à la prime à l'herbe. Par contre, ils bénéficient notablement des nouvelles aides aux cultures : les primes aux céréales représentent la moitié des aides directes perçues par les exploitations laitières spécialisées en 1994.

Pour des raisons symétriques, la progression des aides directes par hectare est moins élevée dans les zones défavorisées et en montagne (respectivement $+83 \%$ et $+51 \%$ sur 1991-94). Ces régions bénéficiaient déjà d'aides substantielles avant réforme : primes bovines liées à la spécialisation viande, indemnités de handicaps, l'impact relatif des nouvelles aides en est donc atténué. De plus, du fait de leurs assolements, les exploitations de montagne bénéficient très peu des nouvelles primes aux cultures.

Notons que, sur la période étudiée, les baisses de prix institutionnels programmées par la réforme ne s'étant pas répercutées sur les prix de marché des produits de l'élevage, la diversité des évolutions de la production par hectare selon les zones renvoie à un faisceau de causes conjoncturelles (aléas de productivité) ou plus permanentes (moindre marge de progrès en zones de montagne).

\section{Les orientations mixtes}

Au sein de cet ensemble, les augmentations 1991-94 de revenu moyen par exploitation sont plus élevées en plaine $(46 \%)$ et en zones défavorisées $(41 \%)$ qu'en montagne $(25 \%)$. Ainsi, les disparités se maintiennent-elles, les revenus moyens des exploitations mixtes des zones défavorisées et de montagne restant nettement plus faibles (graphique 5).

\section{Graphique 5. Ecart à l'EBE moyen national - Orientation "cultures-élevage "}

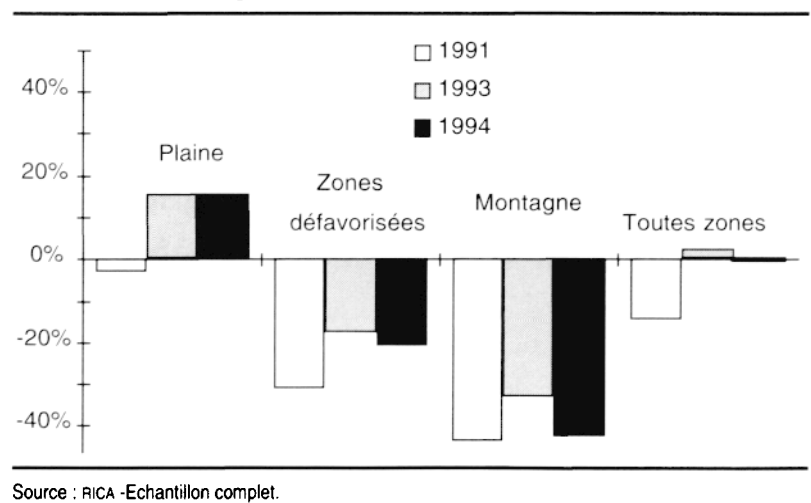


Ces exploitations mixtes sont principalement représentées en plaine et en zones défavorisées. En accédant aux nouvelles primes culturales, les exploitations de plaine bénéficient d'une très forte croissance des aides à l'hectare - elles sont multipliées par quatre - qui compense largement la baisse du produit à l'hectare.

Ceci est encore amplifié par l'agrandissement important de ces exploitations ( $+22 \%$ en SAU), réalisé pour l'essentiel dès 1993. Depuis, le revenu des exploitations mixtes de plaine devient supérieur au revenu moyen national.

En zones défavorisées, on observe des évolutions de même nature - agrandissement, progression des aides et du résultat par hectare -, mais de moindre ampleur. Sous réforme, l'EBE de ces exploitations se rapproche du niveau national, mais lui reste néanmoins inférieur.

En montagne, les orientations mixtes sont assez peu représentées dans le RICA. On note que la progression des aides à l'hectare est plus modérée notamment du fait de l'orientation élevage des exploitations, déjà bénéficiaires de primes importantes avant la réforme.

Après s'être sensiblement rapproché de la moyenne entre 1991 et 1993, le revenu de ces exploitations s'en écarte à nouveau en 1994.

L'évolution des revenus constatée au début de la mise en oeuvre de la réforme (1991-93) se caractérise par un resserrement des disparités de revenu en faveur des zones de montagne et défavorisées. Les nouveaux instruments de la politique agricole concourent pour partie à ce résultat : le "semi-découplage » des aides est plus favorable aux exploitations moins productives; le gel des terres affecte surtout les exploitations de grandes cultures, localisées en plaine; les fortes revalorisations des primes animales profitent principalement aux exploitations d'élevage des régions peu intensives.. Ainsi, en 1993, dans chaque grande orientation de production visée par la réforme, les revenus moyens des différentes zones de handicaps s'étaient rapprochés sensiblement de la moyenne nationale - à l'exception des exploitations mixtes de plaine.

Dès 1994, les écarts se reforment à nouveau dans l'orientation cultures: les exploitations de plaine retrouvent un revenu supérieur de $20 \%$ à la moyenne nationale, après s'en être faiblement rapprochées; en zones défavorisées, l'écart constatée avant réforme se reconstitue presque intégralement. En élevage, par contre, la meilleure situation acquise par les zones défavorisées et de montagne en 1993 perdure en 1994. Si la réforme a permis une réduction des disparités, elle n'a cependant pas modifié la hiérarchie des revenus : les éleveurs bovins et ovins des régions les moins favorisées ont toujours les revenus les plus faibles.

\section{La réduction des inégalités peut-elle être durable?}

\section{Réouverture des écarts de revenu en 1994 et 1995}

Les comptes provisoires par catégorie d'exploitation établis pour l'année 1995 (SCEES, 1995) font apparaître une nouvelle augmentation du revenu brut d'exploitation agricole entre 1994 et $1995(+8,5 \%$ pour le RBE en optique production (4) des exploitations à temps complet).
Graphique 6. Evolution du RBE moyen par OTEX (optique production)

\section{Illustration non autorisée à la diffusion}

\section{Source : SCEES, 1995}

Cette progression est notamment due à celles enregistrées par la viticulture $(+22,1 \%)$ et l'élevage hors-sol $(+21 \%)$, productions plutôt pratiquées en zones de plaine.

Les exploitations de grandes cultures, majoritairement situées en plaine voient leur rbe progresser de $6 \%$ en moyenne. Cette orientation a notamment bénéficié d'une conjoncture favorable pour les prix des céréales $(+2,3 \%$ par rapport à 1994 , malgré la baisse du prix d'intervention), et d'une hausse de $16 \%$ des subventions d'exploitation.

Le revenu des élevages laitiers progresse de $+9 \%$, leurs subventions d'exploitation augmentant en moyenne de $32 \%$, notamment sous l'effet de la montée en puissance des aides compensatrices céréalières, dont bénéficient les exploitations laitières à système fourrager intensif.

L'orientation bovin viande, particulièrement représentée en zones défavorisées, enregistre une baisse de son RBE de $-4,4 \%$, malgré la progression des subventions d'exploitation $(+14 \%)$. Les cours des bovins ont été orientés à la baisse en 1995 (-8\% par rapport à 1994), suite à la diminution des prix d'intervention, programmée par la réforme, et aux dévaluations survenues dans les pays qui s'approvisionnent traditionnellement sur le marché français - Espagne et surtout Italie.

Baisses dans les orientations à faible revenu - bovins viande-, forte progression pour les orientations à revenus plus élevés - grandes cultures, viticulture- : l'année 1995 est donc marquée par un léger accroissement de la dispersion des revenus.

Compte tenu de la répartition géographique des différentes productions, il est très probable que ces récentes évolutions de revenu se traduisent au mieux par une nouvelle atténuation des effets redistributifs de la réforme de la PAC entre les zones de handicaps, voire par un retour aux disparités antérieures.

\section{Projections à l'horizon réforme accomplie}

Il s'agit d'une simulation sommaire qui consiste à appliquer des variations de prix et d'aides directes aux résultats du RICA de 1994, toutes choses égales par ailleurs (programme Pecari, Blogowski, Boyer, 1994).

4. Optique production : compte tenu des variations de stocks, contrairement à l'optique livraisons. L'optique production est donc moins éloignée de l'approche comptable d'entreprise, comme celle du RICA. 
De 1994 à l'achèvement de la réforme de la PAC (campagne 1995-96), les primes compensatrices céréalières et les aides à l'élevage augmenteront, selon des modalités réglementaires connues.

De nouvelles baisses des prix d'intervention sont également programmées. Leur impact effectif sur les prix réellement perçus par les agriculteurs est difficilement prévisible, comme l'ont montré les premières années de la réforme. Nous avons donc fait l'hypothèse d'un maintien des prix observés en 1995 -donnés par les comptes nationaux provisoires.

Les prix de 1995 ont été également appliqués aux produits hors réforme et aux principales consommations intermédiaires.

Les résultats de cette simulation sont résumés ci-dessous :

Tableau 4. Variation relative d'EBE simulée de 1994 à 1996

\begin{tabular}{lrrrr}
\hline & Plaine & Montagne & $\begin{array}{r}\text { Zones } \\
\text { défavorisées }\end{array}$ & Ensemble \\
\hline Cultures & $7 \%$ & - & $8 \%$ & $7 \%$ \\
\hline Elevage & $-1 \%$ & $6 \%$ & $2 \%$ & $2 \%$ \\
\hline Mixtes & $3 \%$ & $6 \%$ & $6 \%$ & $3 \%$ \\
\hline Autres & $11 \%$ & - & - & $10 \%$ \\
\hline Ensemble & $6 \%$ & $6 \%$ & $4 \%$ & $6 \%$ \\
\hline
\end{tabular}

L'augmentation de l'EBE moyen de l'orientation cultures est pratiquement identique en zones de plaine et en zones défavorisées, le léger avantage de ces dernières découlant de l'impact un peu plus important de la revalorisation des aides sur des résultats à l'hectare plus faibles en 1994.

La légère diminution du revenu de l'orientation élevage en plaine est essentiellement due à la progression des charges $(+1 \%)$. La revalorisation des aides à l'élevage est ici limitée, d'une part par les chargements, excluant de nombreuses exploitations de la prime à l'herbe et plafonnant les aides bovines, d'autre part, par l'orientation laitière de nombreux élevages - exclus de fait des primes bovines. Toutefois, les aides supplémentaires compensent intégralement la diminution de produit, au demeurant limitée $(-2 \%)$ du fait du poids des exploitations laitières spécialisées, moins touchées par la baisse des prix des bovins.

En zones défavorisées, le revenu de l'orientation élevage progresse faiblement : le supplément d'aides directes est légèrement supérieur à la baisse du produit et à l'augmentation des charges $(1 \%)$.

L'évolution du revenu de l'élevage est plus favorable en montagne du fait des faibles chargements des exploitations - accès à la prime à l'herbe, pas de plafonnement des primes bovines. En outre, l'impact de la baisse des prix des bovins est ici limitée par la présence des exploitations laitières.

Compte tenu du poids des différentes orientations dans chaque zone, un écart notable de revenu se reconstitue aux dépens des zones défavorisées : l'EBE moyen n'y progresserait que de $2 \%$ contre $6 \%$ en plaine. Les zones de montagne maintiendraient leur position relative.

Ainsi, les nouveaux dispositifs de soutien ont contribué à la réduction des inégalités de revenu lors de leur première année d'application. Par la suite, leur impact mécanique s'atténue et n'entraîne pas un nouveau resserrement de la hiérarchie des revenus entre zones de handicaps.
Par nature, ces projections ne prennent pas en compte les futures adaptations techniques et structurelles des exploitations. Il est probable que les processus d'agrandissement observés antérieurement se poursuivront, les aides directes assises sur les dimensions de l'appareil de production - hectares ou têtes de bétail - jouant dans ce sens. Ces évolutions risquent cependant d'être moins rapides dans les régions plus favorisées, où la démographie agricole et la productivité des terres sont moins favorables à la libération de surfaces.

Nous avions en effet constaté qu'entre 1991 et 1993, les agrandissements des exploitations de grandes cultures étaient sensiblement supérieurs dans les régions de petites terres (diagonale allant du centre-ouest au centre-est) que dans le grand Bassin Parisien (Blogowski, Boyer, 1995).

Par contre, les voies d'amélioration du revenu par les réductions d'intrants sont probablement plus étroites en zones difficiles, les agriculteurs de ces régions ayant déjà été amenés à contenir fortement leurs charges.

L'incitation à l'agrandissement, fournie par les aides, risque en outre de s'atténuer à terme sous l'effet de l'incorporation de la valeur de ces primes dans le prix du foncier (Boussemart, Vermersch. 1993), les perspectives de croissance du revenu en zones difficiles s'en trouveraient alors limitées.

Gilles BAZIN, INRA, INA • Alain BLOGOWSKI, ministère de l'Agiculture - DAFE • Philippe BOYER, ministère de l'Agriculture - DAFE. Décembre 1995.

\section{RÉFÉRENCES BIBLIOGRAPHIQUES}

Bazin G. Le soutien des revenus dans les zones de montagne et défavorisées. Agreste, 1993, Cahiers nº 16.

Bazin G., Carles R., Kroll J.C. Les effets différenciés de la réforme de la PAC dans quatre régions de grande culture. INRA-ESR, Grignon, 1995, étude économique ${ }^{\circ} 20$.

Blogowski A., Boyer $\mathrm{Ph}$. Les effets différenciés de la réforme de la PAC sur les revenus des agriculteurs. Economie Rurale, 1994, n²20-221.

Blogowski A., Boyer Ph., Ronssin B. La réforme de la PAC: les exploitations de grandes cultures s'adaptent. Chambres d'Agriculture, 1994, $n^{\circ} 831$.

Boyer $\mathrm{Ph}$. Les exploitations bovines au début de la nouvelle PAC. Note du Bureau de l'évaluation et de la prospective, Ministère de l'Agriculture et de la Pêche, Direction des Affaires Financières et Economiques, 1995.

Boussemart J. Ph., Vermersch D. L'évolution des rendements céréaliers dans le cadre de la réforme de la PAC : entre inefficacité technique et prix-efficacité. INRA ESR, Ministère de l'Economie - Direction de la Prévision, 1993.

Commission Des Communautes Européennes. Evolution et avenir de la PAC. Com (91) 100 final, 1991.

SCEES. Les comptes prévisionnels par catégories d'exploitations pour 1995 : poursuite de l'amélioration générale des revenus. Agreste, 1995, Données n85. 